\title{
A Novel Method for Field Analysis and Design of Electromagnet Used in Lifting Applications
}

\author{
Ali Hashemi, Parsa Yazdanpanah Gharaei \\ Department of Electrical and Computer Engineering, Faculty of Mohajer, \\ Technical and Vocational University (TVU), Isfahan, Iran \\ *corresponding author, E-mail: ahashemi@tvu.ac.ir
}

\begin{abstract}
Magnetic crane is one of the industrial applications of magnetic absorption, which uses an electromagnet to create the necessary force for lifting and moving objects. In this paper, the field analysis and design of an electromagnet are discussed. During the electromagnet analysis and design process, with special attention to leakage fluxes and fringing effects, a new magnetic equivalent circuit for an electromagnet is proposed. Also, the nonlinear behavior of the $\mathrm{B}-\mathrm{H}$ curve of the ferromagnetic core and its saturation point are considered. Due to the various shapes of the ferromagnetic core, the U_I structure is selected with a symmetrical winding to reduce leakage flux and fringing effects. MATLAB software is used for analyzing and designing the electromagnet, and ANSYS Maxwell software is used to simulate its magnetic field. To verify the accuracy of the proposed method, the prototype was made of a magnetic crane with designed electromagnet. The comparison between measurement and simulation results shows that the error is less than $2 \%$, which confirms the accuracy of the proposed method.
\end{abstract}

Keywords: Electromagnet, Magnetic Equivalent Circuit, Magnetic Field, Leakage Flux, Fringing Effects.

\section{Introduction}

Electromagnet is one of the main component which is used in equipment such as motors, generators, MRI devices, magnetic suspension systems in the Maglev trains, and also in magnetic cranes for lifting and moving objects.

In the textbooks, simple magnetic equivalent circuits are introduced for electromagnet. By analyzing them, the magnetic flux inside the core can be calculated [1]. In this analysis method, usually the leakage flux, fringing effects, nonlinearity relation between $\mathrm{B}$ and $\mathrm{H}$ of the core and its saturation point are not considered. Therefore, the obtained results have low accuracy and are not reliable in practical applications.

In [2], several permeances are added to the simple magnetic equivalent circuit to take into account the leakage fluxes and fringing effects. It does not have field analysis, and the location of some of the permeances are not sufficiently precise.

In [3-5], various structures of ferromagnetic core and their winding location have been investigated and analyzed in terms of leakage flux and fringing effect. The result of this research is that the U_I structure with symmetrical single coil has the lowest magnetic flux losses.
In [6], the finite element method (FEM) is proposed to calculate the electromagnetic force in moving magnetic suspension systems, but nonlinear behavior of the B-H curve and its saturation point are not considered.

This paper, by introducing a precision magnetic equivalent circuit for electromagnet, is intended for field analysis, designing and manufacturing a prototype of an electromagnet based on the design parameters derived from the proposed algorithm. To this end, the proposed magnetic equivalent circuit is initially introduced and analyzed, then an electromagnet is designed. There are many parameters that are effective in design. Therefore, some of them, called "design constants", are known, and the rest, called "design variables", will be calculated by the design algorithm in MATLAB software. To validate the proposed method, the electromagnet is simulated by using ANSYS Maxwell (MAXWELL) software and its results are compared with the design algorithm results. Finally, the electromagnet is made and placed on a suitable platform to measure lifting force.

\section{Electromagnet Analysis}

\subsection{Magnetic Field Study}

Figure 1 shows an electromagnet consisting of N-turn windings with dc current $i$ wrapped on a ferromagnetic core. The U-shaped is fixed but the I-section is movable. In this figure, $\Phi$ is the magnetic flux and $B$ is the magnetic flux density or magnetic field. When the I-section is close to the U-shape core portion, a large part of the field B is placed on the core path. But part of the magnetic flux does not go through the core, which is called leakage flux. The magnetic flux leakage is due to non-zero air permeability. In the air gaps the magnetic field lines are no longer confined by the core, so they 'bulge' out beyond the outlines of the core before curving back to enter the next piece of core material, reducing the field strength in the gap. The bulges are called fringing field.

In Fig. 1, the lines of the leakage and fringing fields, $B_{L}$ and $B_{F}$, are shown. These fields reduce the total magnetic flux in the core and ultimately reduce the electromagnet's lifting force.

In order to have an accurate and efficient analysis method, several factors have to be considered. The first is the leakage flux and its consideration in the analysis method. The second is the edge effect, which causes the magnetic field lines to exit the main path in the air gap. In [1], it is suggested that the effective cross-sectional area of the air gap is slightly larger than the ferromagnetic core cross section and the third one is the hysteresis in ferromagnetic materials. 


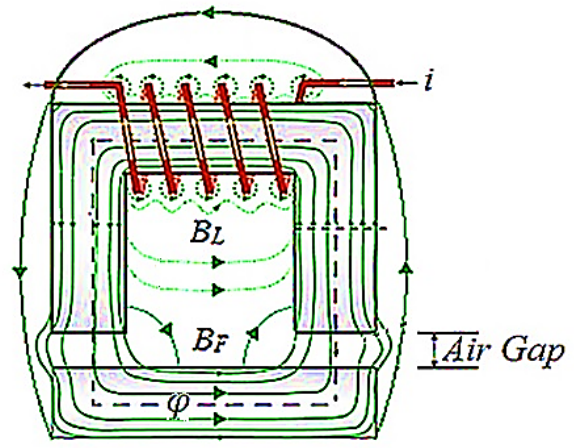

Figure 1: Electromagnet with U_I structure and magnetic flux paths

\subsection{Magnetic Equivalent Circuit}

Figure 2-a shows the partially geometric structure of an electromagnet with a U-I core. The size of the different sections of the core and the coil are marked on the figure. In Fig. 2-b, the magnetic equivalent circuit is proposed for the electromagnet in Fig. 2-a. This circuit is inspired by what is outlined in [2] and is being improved. Permeances for the calculation of leakage fluxes in the magnetic equivalent circuit are: horizontal leakage permeance $P_{h l}$ and vertical leakage permeance $P_{v l}$ of the coil, horizontal leakage permeance under the coil $P_{h b}$, and vertical bypass leakage permeance $P_{v b}$.

The formula for calculating these permeances is presented in (1) to (4).

$P_{h l}=\frac{\mu_{0} d_{w} d}{3 w_{s}}+\frac{2 \mu_{0} d_{w}}{3 \pi} \ln \left(1+\frac{\pi w_{e}}{w_{s}}\right)$

$P_{v l}=\frac{1}{6} \frac{\mu_{0} w_{w} d}{d_{s}+x}$

$P_{h b}=\mu_{0}\left(\frac{\left(d_{s}-d_{w}\right) d}{w_{s}}+2 \frac{\left(d_{s}-d_{w}\right)}{\pi} \ln \left(1+\frac{\pi w_{e}}{w_{s}}\right)\right)$

$P_{v b}=\mu_{0}\left(\frac{\left(w_{s}-w_{w}\right) d}{d_{s}+x}+2 \frac{\left(w_{s}-w_{w}\right)}{\pi} \ln \left(1+\frac{\pi Y}{d_{s}+x}\right)\right)$

In which

$Y=\min \left(w_{b}, w_{i}\right)$

The computational formula of the end leakage permeance, $P_{e}$, is given in [2].

The main permeance of the air gap is:

$P_{G}=\mu_{0} \frac{w_{e} d}{x}$

Permeances caused by edge effects in four aspects of the air gap are:

$P_{f F}=P_{f B}=\frac{\mu_{0} w_{e}}{\pi} \ln \left(1+\frac{\pi w_{i}}{x}\right)$

$P_{f o}=\frac{\mu_{0} d}{\pi} \ln \left(1+\frac{\pi w_{i}}{x}\right)$

$P_{f I}=\frac{2 \mu_{0} d}{\pi} \ln \left(1+\frac{\pi X}{4 x}\right) ; X=\min \left(d_{s} ; w_{s}\right)$

The permeances $P_{f F}, P_{f B}$, and $P_{f O}$ in the air gap are in parallel with $P_{G}$. However, $P_{f I}$ is considered to be $P_{3,5}$ given the operating range in the proposed magnetic equivalent circuit.

$P_{3,5}=P_{6,8}=P_{f I}$

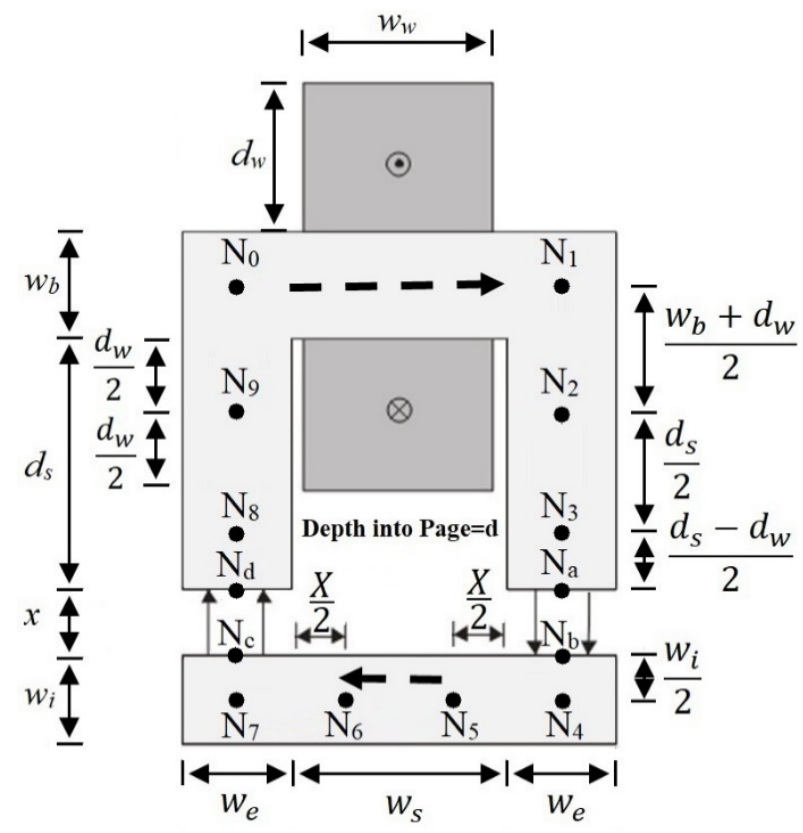

(a)

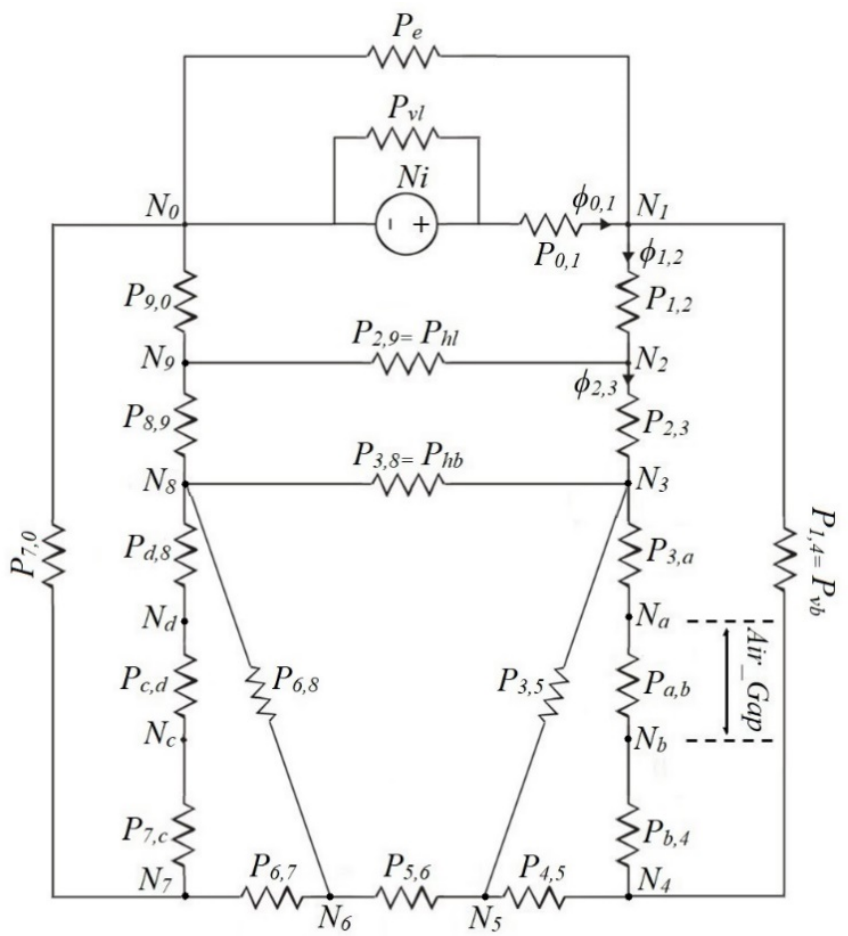

(b)

Figure 2: An electromagnet (a) Structure and Dimensions, (b) Proposed Magnetic Equivalent Circuit

Permeances of the main path of flux are as follows:

$$
\begin{aligned}
& P_{0,1}=\mu \frac{w_{b} d}{w_{s}+w_{e}} \\
& P_{1,2}=P_{0,11}=\mu \frac{2 w_{e} d}{w_{b}+d w} \\
& P_{2,3}=P_{10,11}=\mu \frac{2 w_{e} d}{d_{s}} \\
& P_{3, a}=P_{\mathrm{d}, 8}=\mu \frac{2 w_{e} d}{d_{s}-d_{w}} \\
& P_{a, b}=P_{c, d}=P_{G}+P_{f F}+P_{f B}+P_{f O}
\end{aligned}
$$


$P_{\mathrm{b}, 4}=P_{7, \mathrm{c}}=\mu \frac{2 w_{e} d}{w_{i}}$

$P_{4,5}=P_{6,7}=\mu \frac{2 w_{i} d}{X+w_{e}}$

$P_{5,6}=\mu \frac{w_{i} d}{w_{s}-X}$

Nodal analysis of the circuit leads to a matrix equation in (19).

$[\mathrm{P}](\mathrm{F})=(\Phi)$

In which the $[\mathrm{P}]_{9 \times 9}$ permeance matrix of the circuit, $(\mathrm{F})_{9 \times 1}$, the vector of the magnetomotive force $(\mathrm{mmf})$ of the circuit nodes, and $(\Phi)_{9 \times 1}$ is the vector of the magnetic flux source of the circuit. According to Fig. 2, it is clear that only the first element of the vector $(\Phi)$ is non-zero.

$\Phi_{1}=N i P_{0,1}$

Regarding the nonlinear behavior of the B-H curve, it is clear that $\mu$ is not constant in different sections of the core. To solve this problem, first, by using the interpolation method and using the MATLAB software, the $\mu$ function is determined in relation to $B$, then a successive approximation method is used, that is, the circuit analysis with an initial value for $\mu$ starts, and $B$ in different sections of the core is determined. Subsequently, $\mu$ is obtained in different parts of the core. These values correct the permeances. Then the second approximation for $B$ in different parts of the core is obtained with corrected permeances. By new values of $B$, new permeability and new permeances are determined. This procedure is repeated until subsequent iterations create small changes in the calculated values.

In Fig. 3, the proposed algorithm for electromagnet analysis is presented which is run in MATLAB. The Error vector in this algorithm shows the difference between the new value of $\mu$ and its previous value.

$$
\text { Error }=\left(\mu_{\mathrm{j}}\right)-\left(\mu_{\mathrm{j}-1}\right)
$$

$j=$ number of iteration

When the Error is smaller than a certain value (so small), the calculated fields and permeances are acceptable.

The magnetic energy stored in the inductance of the circuit is equal to:

$W_{m}=\frac{1}{2} L i^{2}$

Where $L$ is the equivalent inductance of the circuit and is expressed by Eq. (23).

$L=N^{2} P_{e q}=N^{2}\left(P_{v l}+P_{T}\right)$

Where $P_{e q}$ is the equivalent permeance seen from the flux source $(\mathrm{Ni})$, and $P_{T}$ is calculated from Eq. (24).

$P_{T}=P_{0,1}\left(1-\frac{F_{1}}{N i}\right)$

For ease of programming, a matrix format is used to calculate the $P_{T}$.

$P_{T}=P_{0,1}\left\{1-\left.\left.P_{0,1}\left[P^{-1}\right]\right|_{1 \text { st row }}[P] P_{0,1}\left[P^{-1}\right]\right|_{1 \text { st column }}\right\}$

Since energy variations are due only to the change in the length

\footnotetext{
${ }^{1}$ Magnesil $=97 \% \mathrm{Fe}+3 \% \mathrm{Si}$
}

of the air gap, the lifting force of the electromagnet can be calculated from Eq. (26):

$$
\boldsymbol{F}_{e}=-\nabla W_{m}=-\boldsymbol{a}_{x} \frac{1}{2} i^{2} \frac{d L}{d x}
$$

In Eq. (26), the minus sign represents the absorbing force. If the electromagnet operates vertically, this force will be able to absorb and lift the mass $m$ :

$m=\frac{F_{e}}{g} \quad(K g)$

Where $g$, the gravity acceleration is equal to $9.8\left(\mathrm{~m} / \mathrm{s}^{2}\right)$.

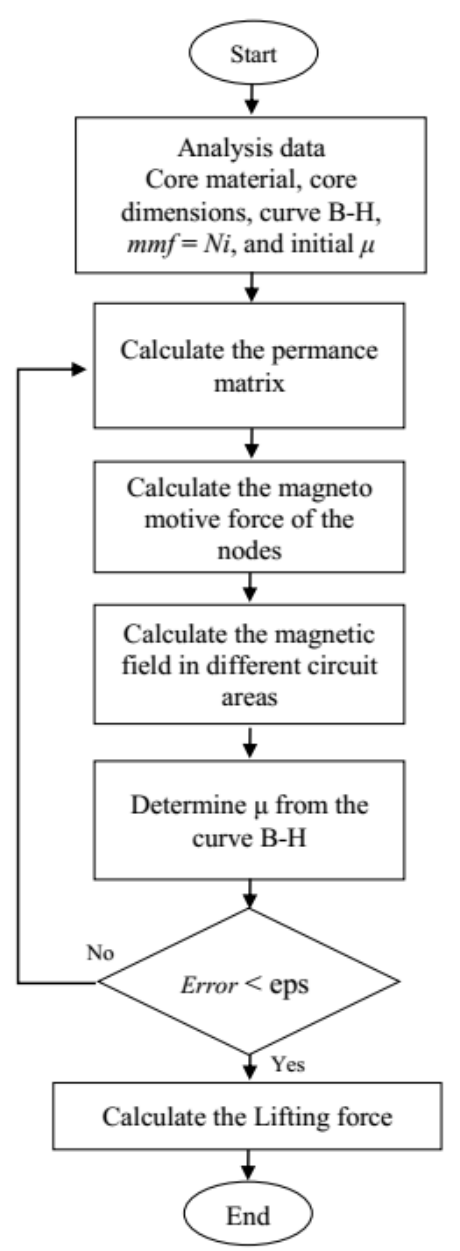

Figure 3: Proposed algorithm for electromagnet analysis

\section{Electromagnet Design}

This section focuses on the design of an electromagnet. Due to the large number of design parameters, some of them are called design constants and are chosen at the beginning of the design. The rest, called design variables, will be calculated during the design process. In the design process, material availability and cost optimization will be considered.

\subsection{Design Constants and Variables}

Design constants used in this section include:

Material of the core - Due to market constraints, the core is selected from the Magnesil ${ }^{1}$ material. In [7], the magnetic properties of this alloy, including the B-H curve and its 
saturation point, are given. The $\mathrm{H}(\mathrm{B})$ function is obtained using the interpolation method by the MATLAB software CFTOOL toolbox according to (28).

$H(B)=\left\{\begin{array}{lc}c_{1} B+c_{0} & ; 0 \leq B<1.39 \\ \frac{10^{7}}{4 \pi}\left(a_{4} B^{4}+a_{3} B^{3}+a_{2} B^{2}+a_{1} B+a_{0}\right) & ; 1.39 \leq B \leq B_{\text {sat }}\end{array}\right.$

Where the coefficients $c_{i}$ and $a_{i}$ are as follows:

$c_{1}=2.823675498094318$

$c_{0}=-39.268139954120301$

$a_{4}=0.308366971026957$

$a_{3}=-1.792388322807561$

$a_{2}=3.906178323475740$

$a_{1}=-3.782445905254492$

$a_{0}=1.372963022261111$

Finally, the function $\mu(\mathrm{B})$ is obtained from relation (29):

$\mu(B)=\frac{1}{d H / d B} \quad ; B \leq B_{\text {sat }}$

Geometric structure of the core - There are many different structures for the core. The E_U, E_I, U_U and U I structures are the most common geometric structures for determining the shape of a ferromagnetic core. In [3-5] using ANSYS software, these structures have been compared with each other in terms of leakage fluxes and fringing effects, and the U_I structure has been recommended as the most suitable type of them. Therefore, in this paper the U_I geometry is used for the core. The mass of the U-shape portion is $m_{U}=0.8438 \mathrm{Kg}$ and the mass of I-shape portion is $m_{I}=0.4219 \mathrm{Kg}$.

Material of the winding - The copper wire is selected with a maximum current density of $7.62 \times 106 \mathrm{~A} / \mathrm{m}^{2}$. The mass density of copper is $8960 \mathrm{Kg} / \mathrm{m}^{3}$.

Location of the winding - In [3], various winding location have been investigated. For having the lowest leakage flux, the proper location of the coil is the horizontal side of the Ushaped core. This configuration gives a symmetrical appearance to the electromagnet.

Load - The propose is to lift a load of $m_{L}=15 \mathrm{Kg}$. During the calculation of the required force, the weight of I-shaped core will also be added to load, that is, $F_{r e q}=m g$ where $m=m_{I}+m_{L}$.

Air gap - The goal is to absorb and lift the load at a distance of $5 \mathrm{~mm}$ from the electromagnet.

Therefore, the structure for design electromagnet is represented in Fig. 2(a). In Tab. 1 the specifications of the core and the coil are presented. With these values, the core cross section is fixed everywhere and equals to $16 \times 36 \mathrm{~mm}^{2}$.

Based on the design constants, the design variables are as bellow:

- DC electric current needed to feed the coil $(i)$.

- Number of turns required for winding $(N)$

- Width of coil $\left(w_{w}\right)$

- Height of coil $\left(d_{w}\right)$

By determining the diameter of the wire, the height, width, and mass of the coil are variables that will be determined during design. Their values must be satisfied in Eq. (30):

$w_{w}<w_{s} ; d_{w}<d_{s}$
Table 1: core and coil specifications

\begin{tabular}{|c|c|c|}
\hline Quantity name & Symbol & Size \\
\hline Slit width & $w_{s}$ & $64 \mathrm{~mm}$ \\
\hline Slit height & $d_{s}$ & $48 \mathrm{~mm}$ \\
\hline Arms width & $w_{e}$ & $16 \mathrm{~mm}$ \\
\hline Width of I- shape core & $w_{i}$ & $16 \mathrm{~mm}$ \\
\hline Width of back of the core & $w_{b}$ & $16 \mathrm{~mm}$ \\
\hline Core thickness & $d$ & $36 \mathrm{~mm}$ \\
\hline Air gap & $l_{g}$ & $5 \mathrm{~mm}$ \\
\hline U-part weight & $m_{U}$ & $0.8438 \mathrm{Kg}$ \\
\hline I-part weight & $m_{I}$ & $0.4219 \mathrm{Kg}$ \\
\hline Width of winding & $w_{w}$ & $\begin{array}{c}\text { Will be } \\
\text { calculated }\end{array}$ \\
\hline Height of winding & $d_{w}$ & $\begin{array}{c}\text { Will be } \\
\text { calculated }\end{array}$ \\
\hline Coil weight & -- & $\begin{array}{c}\text { Will be } \\
\text { calculated }\end{array}$ \\
\hline
\end{tabular}

\subsection{Design Algorithm}

The design algorithm is shown in Fig. 4. To calculate the design variables, at first, lifting force must be calculated as $F_{e}=m g$.

Due to the saturation in the ferromagnetic core, there is a limitation of the maximum force applied to the core $[8,9]$. The maximum force is given by Eq. (31).

$F_{\text {Max }}=2 \times \frac{B_{\text {sat }}^{2} \times S}{2 \mu_{0}}$

Thus, with the specific structure of the core and the desired force, if $F_{e}$ is greater than $F_{\text {Max }}$, then a larger core must be used. After assuring that the core is not saturated, the required magnetic field in the air gap $\left(B_{\text {gap }}\right)$ is calculated using Eq. (32):

$B_{g a p} \approx \sqrt{\frac{\mu_{0} F_{e}}{S}}$

Then the initial value for $\mu$ and the diameter of the wire is selected. Withdrawal of leakage fluxes and fringing effects, the magnetomotive force is calculated by Eq. $(33)[10,11]$.

$N i \approx 2 x \sqrt{\frac{F_{e}}{\mu_{0} S}} \quad(A t)$

This value is selected as the initial value of the required $m m f$ for the electromagnet. The width and the winding height are determined by Eq. (30). By nodal analysis, the $B$ field is determined in different parts of the circuit. The new value of $\mu$ will be calculated by Eq. (29).

By using a procedure of successive approximation, results are acceptable when the difference between the new and previous values of $\mu$ is very small. Now, from the calculated value of the magnetic field in the air gap $\left(B_{g}\right)$, the $m m f$ of the circuit is corrected according to Eq. (34).

$(N i)_{n e w}=(N i)_{o l d}\left(\frac{B_{g a p}}{B_{g}}\right)$

Errors $E_{1}$ and $E_{2}$ indicate the difference between the estimated value and the required value for $\mu$ and $B_{g a p}$ and are determined from the Eqs. (35) and (36):

$E_{1}=\frac{\mu_{i}-\mu_{i-1}}{\mu_{i}}$ 
$E_{2}=\frac{B_{g a p}-B_{g, i}}{B_{g a p}}$

$i=$ Number of iteration

After executing the design algorithm, the final value of $N i=3600$ (At) was determined. To compute the values of $N$ and $i$, it is important to note few points:

- The multiplication of $N$ and $i$ is equal to the magnitude of the magnetomotive force ( $\mathrm{mmf}$ ).

- The number of rounds per layer, as well as the number of winding layers, must be such that the relations in Eq.9 are satisfied.

- The diameter of the wire should be chosen in such a way as to endure the electric current.

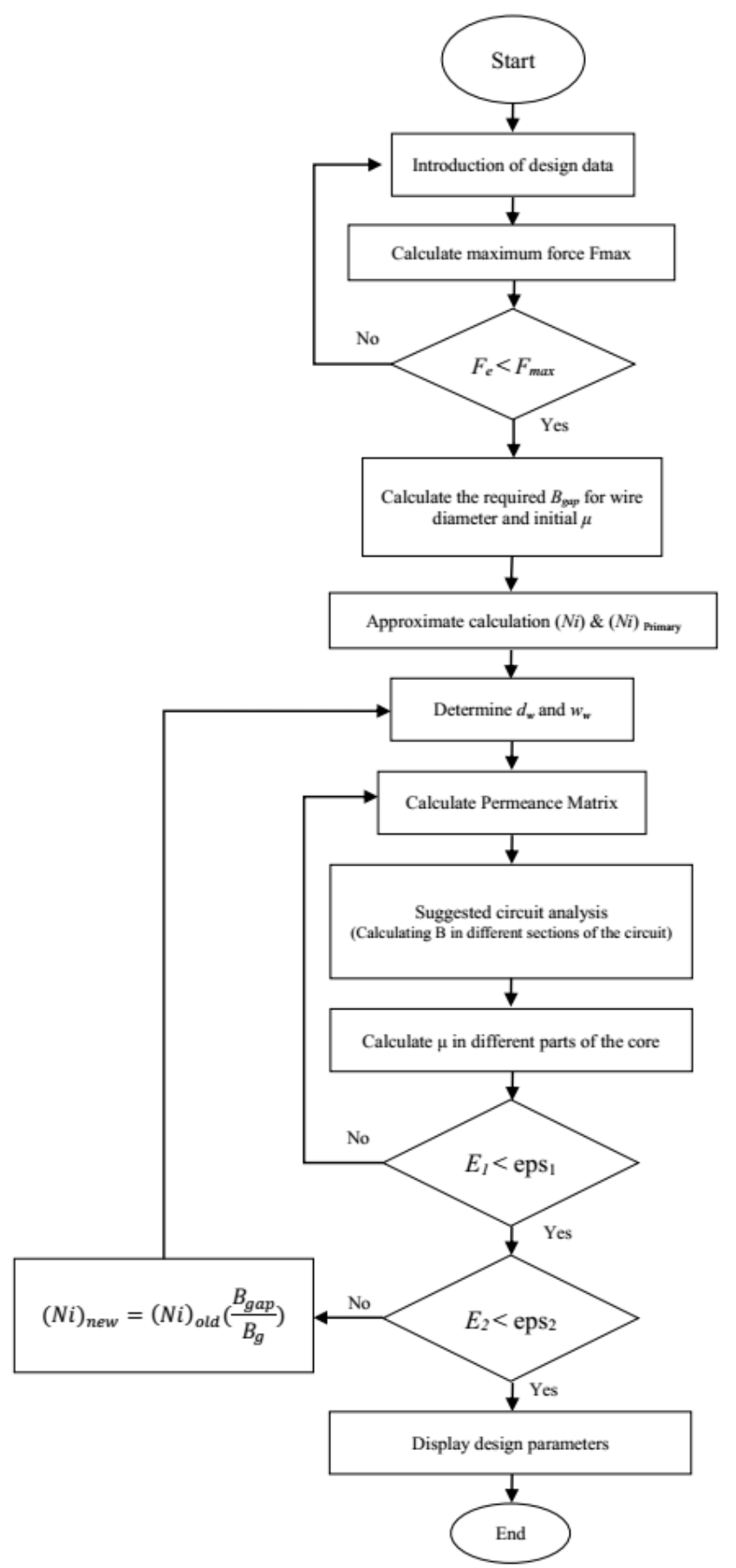

Figure 4: Proposed design algorithm
Under these conditions, the diameter of the wire is equal to 1.2 $\mathrm{mm}$, the electric current is $i=6 \mathrm{~A}$, and the number of winding turn is equal to $N=600$, which is wound in 50 turns per layer. In this case, $d_{w}=14.4 \mathrm{~mm}, w_{w}=60 \mathrm{~mm}$, and the mass of winding is $0.87 \mathrm{Kg}$.

\section{Simulation Results}

Figure 5 shows the structure of the 3D electromagnet designed in the MAXWELL software. The magnetic material, dimensions, and other specifications of the electromagnet are presented in Section 3. In Fig. 6, the simulated magnetic field inside the core and its surrounding environment are represented.

As shown in Fig. 6, the field under the winding inside the core has the highest value. This value is about $1.5 \mathrm{~T}$, which is less than the saturation point of Magnesil's B-H curve. In other parts of the core, some of the flux and magnetic field leaks or loses.

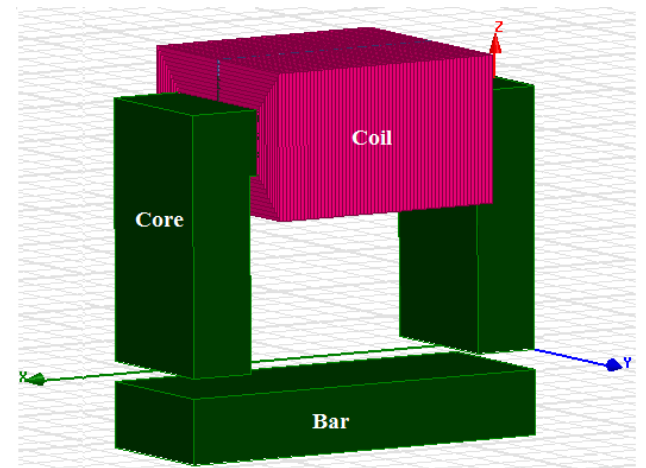

Figure 5: Electromagnet structure in 3D Maxwell Software
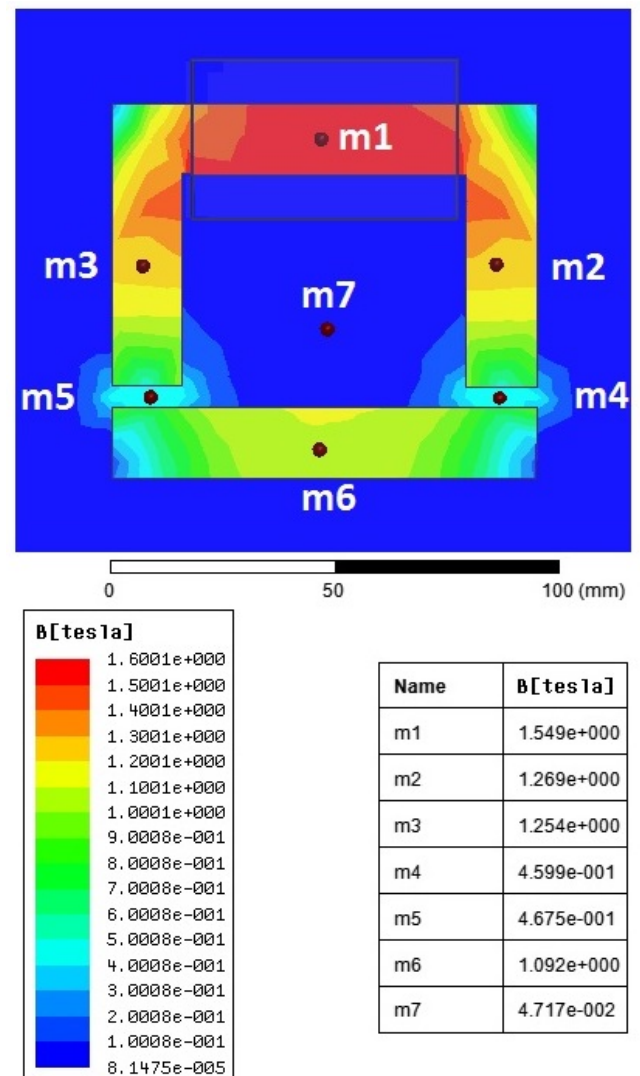

Figure 6: Magnetic field in the electromagnet 
The resultant value of the magnetic fields in the air gap caused by the main path field, leakage and fringing effects is about 0.65 T. In Fig. 6, the main path field is only measured and shown. In this simulation, the lifting force was found to be 144.34 $\mathrm{N}$ and sufficient to lift the load of $14.7286 \mathrm{Kg}$.

The analysis with MATLAB software shown that lifting force of the electromagnet is equal to $145.1 \mathrm{~N}$, which is close to the simulated value and has an error about $0.5 \%$. These results refer to low losses and the suitability of the proposed electromagnetic structure, and also refer to the accuracy of the proposed method.

Figure 7 shows the variation of the lifting force versus length of the air gap. It can be seen that by reducing the air gap, the lifting force increases. Therefore, by adjusting the length of the air gap, it can lift various loads. For example, if the air gap is reduced to $3 \mathrm{~mm}$, the lifting force will be increased to $368 \mathrm{~N}$, which has the ability to lift a mass of 37.5 $\mathrm{Kg}$. On the other hand, if the length of the air gap is increased to $7 \mathrm{~mm}$, the lifting force will be reduced to $76 \mathrm{~N}$, which has the ability to lift a mass of $7.75 \mathrm{Kg}$. The physical justification is that, by reduction of the air gap length, the fringing fields are reduced. So the magnetic field into the I-section of the core is increased. Since the lifting force is proportional to the square of the density of magnetic field, so heavier loads can be lifted and displaced.

In Fig 7, MATLAB and MAXWELL results are compared. This comparison shows a good agreement between results.

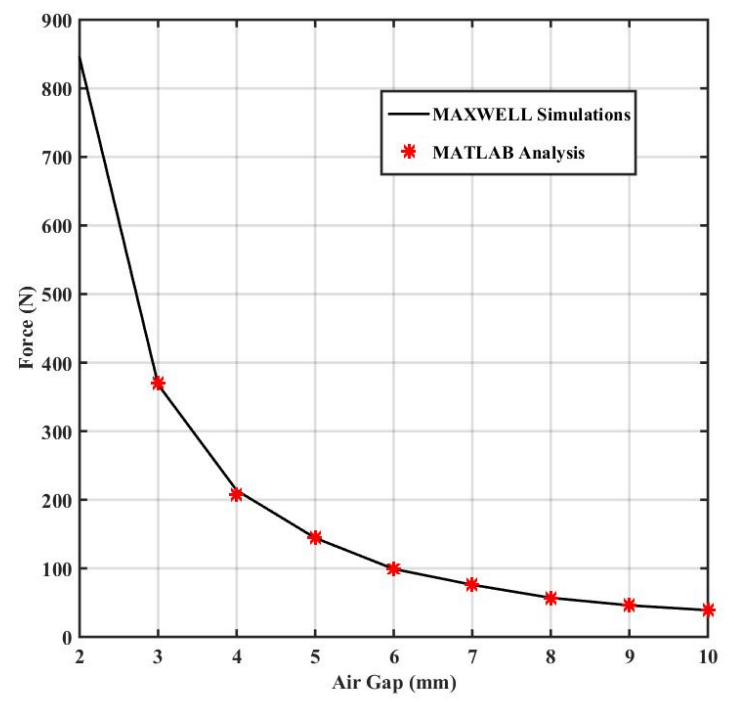

Figure 7: Variations of lifting force with length of the air gap

\section{Experimental Result}

Fig. 8 shows the fabricated laboratory sample of the magnetic crane. The electromagnet used in this crane is based on the information in section 3 . The core of this electromagnet is made of sheets with $0.5 \mathrm{~mm}$ thickness, which are fixed together. The core thickness is $36 \mathrm{~mm}$ and its cross-sectional area is $36 \times 16 \mathrm{~mm}^{2}$ in whole parts of the core. The coil consists of $86.4 \mathrm{~m}$ copper wire with $1.2 \mathrm{~mm}$, wrapped in 12 layers. Each layer is made of 50 turns of winding, which creates a total of 600 turns of coil with 6 A current. The weight of the U-section with the coil is $1.7138 \mathrm{Kg}$, and the weight of the coil is $0.87 \mathrm{Kg}$. The I-section has a weight of $0.4219 \mathrm{Kg}$ and a dimension of $96 \times 16 \times 36 \mathrm{~mm}^{3}$. The crane has two DC electrical motors used for vertical and horizontal moving of the load on the rails which are mounted in the crane's desk. The electrical input power is $12 \mathrm{~V}, 20 \mathrm{~A}$ that supplies the motors and electromagnet's coil. An ultrasonic sensor is also used to measure the air gap length.

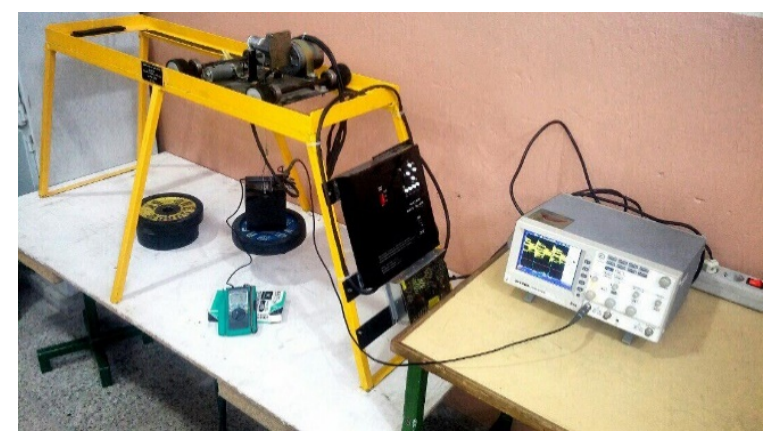

Figure 8: The fabricated magnetic crane

In the tests, the heaviest load that was lifted by the crane from a distance of $5 \mathrm{~mm}$ is equal to $14.5 \mathrm{Kg}$, representing a force equivalent to $142.1 \mathrm{~N}$. The results of measurement have a good agreement with the simulation and MATLAB analysis results. Comparing these results with design values shows a higher than $97 \%$ accuracy. The electromagnet results are listed in Tab. 2.

Table 2: Electromagnet results for air gap $=5 \mathrm{~mm}$

\begin{tabular}{|c|c|c|c|c|}
\hline \multicolumn{2}{|c|}{$\begin{array}{c}\text { MATLAB } \\
\text { Analysis Results }\end{array}$} & \multicolumn{2}{c|}{$\begin{array}{c}\text { MAXWELL } \\
\text { Simulation Results }\end{array}$} & $\begin{array}{c}\text { Measurement } \\
\text { Results }\end{array}$ \\
\hline Fe (N) & $\mathrm{B}_{\text {gap }}(\mathrm{T})$ & Fe (N) & $\mathrm{B}_{\text {gap }}(\mathrm{T})$ & Force (N) \\
\hline 145.1 & 0.6554 & 144.34 & 0.65 & 142.1 \\
\hline
\end{tabular}

\section{Conclusion}

In this paper the novel method for designing and analyzing an electromagnet and its construction were presented. There are many design parameters, thus they were divided into two categories: fixed (known) and variable (unknown). Different geometrical structures of the ferromagnetic core were studied. The field analysis shown that the U_I structure with symmetrical single-winding due to its low leakage flux and fringing field is as a proper structure in this design. According to the available Iranian's magnetic market and technical considerations, the 'Magnesil' was selected as a magnetic laminated core for this propose. Also, considering the maximum weight to lift, the required lifting force was determined. Variables such as wire diameter, electric current, and winding number were determined based on the type and the maximum current density of wire. Finally, the electromagnet was built and tested on it. The experimental results with a precision of over $97 \%$ are close to the simulation and design results, so indicating the accuracy of the proposed method.

\section{Reference}

[1] D. K. Cheng. "Static Magnetic Fields" in Field and Wave Electromagnetics, Massachusetts, USA: Addison Wesley, 1983, pp. 220-230. 
[2] J. Cale, S. D. Sudhoff, and Li-Quan Tan, “Accurately Modeling EI Core Inductors Using a High-Fidelity Magnetic Equivalent Circuit Approach," IEEE Trans. Magnetics, vol. 42, no. 1, pp. 40-46, Jan. 2006.

[3] P. K. Biswas and S. Banerjee, "ANSYS Simulation Based Comparative Study between Different Actuators and Guide-ways used in DC Electromagnetic Suspension Systems," International Journal on Electrical Engineering and Informatics, vol. 4, no. 2, pp. 217-230, July 2012.

[4] S. Banerjee, P. K. Biswas, R. Bhaduri, P. Sarkar, "A Comparative Study between Different Structures of Rail and Actuator Used in Electromagnetic Levitation Systems," in Proc. PEMD, 2010, pp. 954-959, UK.

[5] P. K. Biswas, S. Bannerjee, "Analysis of U_I and U-U Type Rail and Actuator Used in Electromagnetic Levitation System Using FEM Software," International Journal of Emerging Technology and Advanced Engineering, vol. 2, Issue 5, May 2012.

[6] Z. Hederic, D. Sostaric, G. Horvat, "Numerical Calculation of Electromagnetic Forces in Magnetic Actuator for Use in Active Suspension System for Vehicles," Technical Gazette, vol. 20, no. 1, pp. 73-77, Feb. 2013.

[7] Colonel WM. T. McLyman, Transformer and Inductor Design Handbook. New York, USA: Marcel Dekker, Inc., $3^{\text {rd }}$ ed. 2004, pp. 53-54.

[8] Y. Bakhvalov, V. Grechikhin, O. Kravchenko, A. Yufanova, "Optimal Design of Shell-Type Electromagnets of XY-Coordinate Electric Actuator," in Proc. ICPDS, 2016, pp. 94-97, Russia.

[9] L. Kolimas and P. Sul, "Computer Design of Electromagnets," in Proc. EIIC, 2015, pp. 211-214, Poland.

[10] J. Y. Choi, S. Y. Sung, S. M. Jang, S. H. Lee, "Design and Dynamic Analysis of Magnetically Levitated Electromagnets with Low-Resolution Position Sensor," IEEE Trans. Magnetics, vol. 48, no. 11, pp. 4546-4549, Nov. 2012.

[11] J. D. Wise, "Magnetic Devices II: Reluctance and Inductance," Internet:

www.ece.rice.edu/ jdw/435/book/ch9.pdf, Aug. 9, 2011. 